DOI: https://doi.org/10.11144/Javeriana.upsy17-2.aree

\title{
Afrontamiento y regulación emocional en estudiantes de fisioterapia*
}

\section{Coping and Emotional Regulation among Physiotherapy Undergraduates}

Recepción: 15 Enero 2016 | Aprobación: 20 Agosto 2017

\author{
Ramón G. CaBAnach \\ Universidad de A Coruña, España \\ Antonio Souto-Gestal ${ }^{\mathrm{a}}$ \\ Universidad de A Coruña, España \\ ORCID: http://orcid.org/0000-0002-7332-1599 \\ Luz GonzÁlez Doniz \\ Universidad de A Coruña, España \\ TANia Corrás VÁzQuez \\ Universidad de Santiago de Compostela, España
}

\footnotetext{
a Autor de correspondencia. Correo electrónico: antonio.souto@udc.es

Para citar este artículo: Cabanach, R. G., SoutoGestal, A., González Doniz, L., \& Corrás, T. (2018). Afrontamiento y regulación emocional en estudiantes de fisioterapia. Universitas Psychologica, 17(2), 1-13. https://doi.org/10.11144/Javeriana.upsy17-2. aree
}

\section{RESUMEN}

Esta investigación tuvo un doble objetivo: la identificación de distintos perfiles de regulación emocional y la comprobación de si estos perfiles se diferencian en el uso de estrategias de afrontamiento ante el estrés académico. Participaron 487 estudiantes de fisioterapia (74\% mujeres) de distintas universidades, con edades comprendidas entre 18 y 40 años. Se aplicó la Escala de Dificultades en la Regulación Emocional (Difficulties in Emotion Regulation Scale [DERS]) y la escala de Estrategias de Afrontamiento del Cuestionario de Estrés Académico (CEA). Se obtuvo un total de tres perfiles de regulación emocional en el análisis de conglomerados. El primero está compuesto por estudiantes con una baja regulación emocional, un segundo grupo con resultados mixtos y un tercer conglomerado que muestra una alta regulación emocional. Se encontraron diferencias estadísticamente significativas en el uso del total de estrategias de afrontamiento entre los perfiles de baja y alta regulación emocional.

\section{Palabras clave}

estrés académico; estrategias de afrontamiento; regulación emocional; inteligencia emocional.

\begin{abstract}
This paper aims to identify different profiles of emotional regulation, and to see if they differ in academic stress coping strategies. The participants were 487 physiotherapy students (74\% female) from different universities, aged between 18 and 40 years. The Difficulties in Emotion Regulation Scale (DERS) and Cuestionario de Estrés Académico (CEA) were applied. A total of three emotional regulation profiles were obtained in the cluster analysis. The first one is composed of students with low emotional regulation, a second group with mixed results was obtained and a third cluster shows students with high emotional regulation. Statistically significant differences in all the coping strategies were found between the profiles of low and high emotional regulation.
\end{abstract}


Keywords

academic stress; coping strategies; emotional regulation; emotional intelligence.

\section{La inteligencia emocional y las estrategias de afrontamiento ante el estrés}

Los estudios sobre inteligencia emocional han puesto de manifiesto que los individuos con alta inteligencia emocional presentan una mayor capacidad para manejar sus emociones en situaciones de estrés, evitando la rumiación sobre los eventos negativos y permitiéndoles establecer eficazmente sus metas (Salovey, Bedell, Detweiler, \& Mayer, 2000). A nivel fisiológico, las personas con elevadas habilidades emocionales perciben los estímulos estresantes como menos amenazantes, de manera que sus niveles de cortisol y de presión sanguínea resultaban más bajos (Mikolajczak, Roy, Luminet, Fillée, \& de Timary, 2007; Salovey, Stroud, Woolery, \& Epel, 2002). Esta misma relación entre inteligencia emocional y estrés se ha encontrado en diferentes trabajos con estudiantes de distintas titulaciones de ciencias de la salud (p. ej., Birks, McKendree, \& Watt, 2009; Naidoo \& Pau, 2008).

Dentro del constructo global de la inteligencia emocional, la regulación de las emociones es considerada un componente esencial (Austin, Saklofske, \& Mastoras, 2010; Mikolajczak, Nélis, Hansenne, \& Quoidbach, 2008), ya que posibilita la moderación de las emociones negativas y la potenciación de las emociones positivas, proporcionando de este modo un mecanismo adaptativo a los estudiantes, con el fin de que estos se enfrenten más eficazmente a los inevitables factores estresantes que conlleva la vida académica. Debido a su importante papel sobre el bienestar psicológico, la regulación emocional ha sido identificada como un recurso relevante en el afrontamiento de la vida académica (Fischer, Forthun, Pidcock, \& Dowd, 2007).

En el ámbito universitario, en los últimos años han surgido nuevas líneas de investigación que intentan analizar el papel que desempeñan diferentes características psicológicas o recursos intrapsíquicos como protectores del estrés entre estudiantes de titulaciones de ciencias de la salud (Cabanach, Souto-Gestal, FernándezCervantes, \& Freire, 2011; Cabanach, SoutoGestal, \& Franco, 2016; Doherty \& Nugent, 2011; Gibbons, Dempster, \& Moutray, 2011; Pau et al., 2007).

Entre estos recursos intrapsíquicos, el estudio de las competencias relacionadas con el manejo y regulación de las emociones en estudiantes de ciencias de la salud se constituye recientemente en un tópico de gran interés (Birks et al., 2009; Hernandez, Blavo, Hardigan, Pérez, \& Hage, 2010; Por, Barriball, Fitzpatrick, \& Roberts, 2011; Thomas, Cassady \& Heller, 2017). En concreto, parece aceptado que la relación entre inteligencia emocional y el estrés académico se encuentra estrechamente influenciada por los procesos de afrontamiento.

Algunos de los más actuales enfoques explicativos del afrontamiento se apoyan en los resultados de la investigación en emociones, relacionando muy íntimamente los constructos de inteligencia emocional y afrontamiento (Folkman \& Moskowitz, 2004; Skinner \& Zimmer-Gembeck, 2007). En esta línea, la regulación emocional se ha vinculado con un afrontamiento más adaptativo en estudiantes (Downey, Johnston, Hansen, Birney, \& Stough, 2010; Mikolajczak et al., 2008; Petrides, PérezGonzález, \& Furnham, 2007; Saklofske, Austin, Galloway, \& Davidson, 2007; Thomas et al., 2017), lo que conlleva el desarrollo de niveles inferiores de estrés y burnout (Pau et al., 2007). Asimismo, elevados niveles de regulación emocional correlacionan negativamente con el empleo de afrontamiento de tipo desadaptativo (Austin et al., 2010; Saklofske et al., 2007; MacCann, Fogarty, Zeidner, \& Roberts, 2011).

\section{El presente estudio}

Gratz y Roemer (2004) consideran dentro de la regulación emocional diversas dimensiones que permiten su conceptualización: la atención y conciencia de las emociones, la claridad o comprensión de las emociones, la aceptación 
de las emociones, la capacidad para controlar las conductas impulsivas y comportarse de acuerdo con los objetivos deseados cuando se experimentan emociones negativas, así como la capacidad para utilizar estrategias de regulación emocional contextualmente apropiadas para modular las respuestas emocionales con la finalidad de satisfacer los objetivos individuales y las demandas situacionales.

Dentro de las dimensiones que integran la regulación emocional, una de las más estudiadas ha sido la claridad emocional, que se ha relacionado reiteradamente con la búsqueda de apoyo social y especialmente con el afrontamiento social (apoyo social y emocional) $y$, en general, con el uso de estrategias de afrontamiento de tipo instrumental (MontesBerges \& Augusto, 2007). Pero se hace preciso profundizar en el conocimiento del papel que desempeña cada una de estas dimensiones en el afrontamiento.

Si bien estas dimensiones están presentes en distinta medida en todos los individuos, consideramos que la combinación que exista entre ellas va a tener relevancia en la gestión diferencial del mundo emocional, es decir, en la regulación emocional. En relación con la inteligencia emocional se han encontrado perfiles diferenciales según la combinación de sus componentes y especialmente en función del factor percepción o atención emocional (Diaz-Castela et al., 2013; Fernández-Berrocal, Extremera, \& Palomera, 2008; Rey, Extremera, \& Pena, 2011; Salguero, Palomera, \& FernándezBerrocal, 2012). Por tanto, puede afirmarse que no todas las dimensiones que integran este constructo inciden de la misma manera en el bienestar del individuo, lo que sugiere la existencia de perfiles diferenciales (GarcíaFernández et al., 2016; Gázquez, PérezFuentes, Díaz-Herrero, García-Fernández, \& Inglés, 2015).

Sin embargo, no se cuenta con datos que permitan afirmar con rotundidad qué combinación de estas dimensiones es la que posibilita un afrontamiento más adaptativo, especialmente en el ámbito universitario. Por eso, esta investigación tiene una triple relevancia: teórica, aportando luz sobre cómo se combinan estas dimensiones en los estudiantes universitarios; metodológica, aportando información sobre cuáles son los perfiles de regulación emocional que pronostican un afrontamiento adaptativo; práctica, aportando información en la línea de desarrollar programas de prevención del estrés en contextos académicos.

Tal y como se ha señalado anteriormente, esta gestión de las emociones adquiere una especial importancia en el afrontamiento del estrés académico, en un contexto de una formación universitaria específica como la de fisioterapia, que conlleva unas condiciones altamente estresantes, caracterizadas por un amplio plan de estudios teórico-práctico y una temprana inmersión en el ámbito clínico (Walsh, Feeney, Hussey, \& Donnellan, 2010).

El presente trabajo se plantea como objetivo preliminar la identificación y caracterización de los perfiles de regulación emocional en una muestra de estudiantes de fisioterapia. Una vez establecida la manera como estos estudiantes combinan las dimensiones que componen la regulación emocional originando perfiles específicos, el objetivo principal será determinar si existen diferencias en el uso de estrategias de afrontamiento frente al estrés académico.

La hipótesis de que ciertos perfiles de regulación emocional podrían resultar más adaptativos que otros ante las situaciones estresantes, traduciéndose en una utilización más eficaz de las diferentes estrategias de afrontamiento activo, parece de vital importancia a la hora de optimizar los programas de prevención del estrés académico.

\section{Método}

\section{Participantes}

La muestra utilizada estaba integrada por 487 estudiantes de los tres primeros cursos de la titulación de Fisioterapia de diversas universidades españolas de las comunidades 
autónomas de Galicia, Andalucía y Madrid. La edad de los participantes osciló entre 18 y 40 años, siendo la media de edad de 21.06 y la desviación estándar de 3.74. Del total de la muestra, el $74 \%$ estuvo compuesto de mujeres y el $26 \%$, de varones, lo que supone una representación bastante fiel de la habitual distribución por sexo en esta titulación, donde existe una clara mayoría femenina (Walsh et al., 2010).

Los sujetos fueron seleccionados a partir de un muestreo probabilístico por conglomerados de una etapa, con el fin de respetar el agrupamiento natural de los estudiantes. Por ello, se consideró como conglomerado cada grupo clase, integrado por aquellos estudiantes que voluntariamente colaboraron en la cumplimentación de los cuestionarios (muestra aceptante).

\section{Instrumentos}

Escala de Dificultades en la Regulación Emocional (Difficulties in Emotion Regulation Scale [DERS]; Gratz \& Roemer, 2004; versión española de Hervás \& Jódar, 2008)

La adaptación española de Hervás y Jódar (2008) usada en el presente estudio redujo a 28 el número de ítems de la escala original, que constaba de 36 ítems con un formato de respuesta tipo Likert de 5 niveles $(1=$ Casi nunca; 5 = Casi siempre), en la que el estudiante indicó la frecuencia con la que experimenta diferentes dificultades en relación con sus experiencias emocionales. Las dimensiones Dificultades en el control de impulsos y Acceso limitado a estrategias de regulación, presentes en el instrumento original, son fusionadas en un único factor, compuesto por 9 ítems y redenominado Descontrol emocional. Los restantes cuatro factores son los de Rechazo emocional (7 ítems), Interferencia cotidiana (4 ítems), Desatención emocional (4 ítems) y Confusión emocional (4 ítems). Por tanto, la versión española de la DERS se compone de cinco factores coherentes, que explican un $63.5 \%$ de la varianza, presentando una fiabilidad idéntica a la del instrumento original $(\alpha=0.93)$.
Siguiendo las recomendaciones de los autores de la adaptación española de la DERS (Hervás \& Jódar, 2008) y tras la publicación de algunos trabajos que cuestionaban la dimensionalidad del instrumento en diferentes contextos socioculturales (Bardeen, Fergus, \& Orcutt, 2012; Marín, Robles, González-Forteza, \& Andrade, 2012; Medrano \& Trógolo, 2014), se consideró apropiado tratar de replicar la estructura factorial obtenida, en especial para contrastar la idoneidad de la integración de las dos dimensiones señaladas.

Este estudio de la dimensionalidad de la DERS muestra la misma estructura factorial de cinco factores obtenida por Hervás y Jódar (2008), que dan cuenta de un $64.95 \%$ de la varianza total. Tanto la versión original como la adaptada de la DERS poseen una excelente consistencia interna $(\alpha=0.93)$.

En este trabajo, las distintas dimensiones se han redenominado en sentido positivo, tal y como se justifica en Cabanach et al. (2011), así: Control emocional, Aceptación emocional, Facilitación emocional, Atención emocional y Claridad emocional. En la presente investigación, se han utilizado cuatro de los cinco factores de la DERS: Control emocional, Aceptación emocional, Claridad emocional y Atención emocional, ya que representan los dos grandes componentes de la regulación emocional, el estratégico y el experiencial, que mantienen una vinculación jerárquica pero relacionada. De este modo, la Atención emocional indica la habilidad para percibir estados emocionales (ítem 2: "Presto atención a cómo me siento" o ítem 7: "Doy importancia a lo que estoy sintiendo"), mientras que la Claridad emocional (ítem 8: "Estoy confuso sobre lo que siento") refleja el reconocimiento y comprensión de los mismos. Por otra parte, la Aceptación emocional (ítem 10: "Cuando me siento mal, me da vergüenza sentirme de esa manera") muestra la capacidad del individuo para tolerar estados emocionales negativos sin experimentar secundariamente rechazo o distrés adicional. Finalmente, el Control emocional (ítem 26: "Cuando me siento mal, pierdo el control sobre mi comportamiento") constituye la dimensión 
que implica un mayor nivel de complejidad, ya que agrupa las habilidades para el control de las emociones negativas y para el empleo eficaz de estrategias de regulación emocional contextualmente apropiadas.

Escala de Afrontamiento (ACEA) del Cuestionario de Estrés Académico (CEA) (Cabanach, Valle, Rodríguez, Piñeiro, \& Freire, 2010)

La Escala de Afrontamiento (ACEA) se compone de un total de 23 ítems que intentan medir en qué grado el estudiante emplea determinadas estrategias de afrontamiento tanto cognitivas como conductuales, para dar respuesta al estrés académico. Las respuestas a cada uno de los ítems se realizan sobre una escala tipo Likert de 5 puntos ( $1=$ Nunca; $5=$ Siempre $)$, en la que el estudiante indica la frecuencia con la que utiliza determinadas estrategias de afrontamiento activo. Agrupa tres factores, que explican el $60.98 \%$ de la varianza total. Estos tres factores son los de Reevaluación positiva (ítem 6, "Cuando me enfrento a una situación problemática durante los exámenes, procuro pensar que soy capaz de hacer las cosas bien por mí mismo), Búsqueda de apoyo social (ítem 8, "Cuando me enfrento a una situación problemática busco consejo y solicito ayuda a otras personas) y Planificación y gestión de recursos personales (ítem 11, "Cuando me enfrento a una situación problemática, priorizo las tareas y organizo el tiempo").

En estudios precedentes (p. ej., Cabanach et al., 2010), esta escala mostró una buena consistencia interna, con un valor a de Cronbach para el total de la escala de 0.89 , con valores comprendidos entre 0.84 y 0.91 para los tres factores. Los valores de consistencia interna obtenidos con los datos del presente estudio son también excelentes, con un valor a igual a 0.92 para el conjunto de la escala y para cada uno de los tres factores señalados, entre 0.86 y 0.9 .

\section{Procedimiento}

El estudio se llevó a cabo siguiendo un diseño ex post facto prospectivo simple de corte transversal.
Se procedió a la recogida de los datos relativos a las variables objeto de análisis, en cada uno de los centros a los que asistían los estudiantes que participaron en la investigación. La recolección de información fue realizada mediante la técnica de cuestionario. Estos fueron aplicados individualmente, sin límite de tiempo y en un único momento temporal, por personal especializado que colaboró en el estudio. A los participantes, se les recalcó la importancia de responder con sinceridad a todas las cuestiones planteadas. Asimismo, se les informó del carácter totalmente confidencial de dicha información.

\section{Análisis estadístico}

Con el propósito de identificar los perfiles de regulación emocional de los estudiantes de la muestra, se utilizó el análisis de conglomerados (método quick cluster analysis), que consiste en un método no jerárquico y de reasignación. Este análisis de conglomerados parece un procedimiento adecuado para el establecimiento de patrones en una muestra amplia de sujetos (Hair, Anderson, Tatham, \& Black, 1998; Pastor, Barron, Miller, \& Davis, 2007).

Los patrones de regulación emocional se han definido a partir de las diferentes combinaciones de las cuatro dimensiones de regulación emocional evaluadas por la DERS. Para eliminar el efecto debido a las diferencias en la medida de estas dimensiones (el número de ítems de cada una de los factores es diferente), se llevó a cabo el análisis de conglomerados después de haber estandarizado las puntuaciones directas. El criterio seguido en la elección del número de conglomerados fue el de maximizar las diferencias interclústeres con el fin de conseguir establecer el mayor número posible de grupos con diferentes combinaciones de dimensiones emocionales.

Para conocer las posibles diferencias entre los patrones respecto a la utilización de las distintas estrategias de afrontamiento activo, se recurrió al análisis de varianza. Dado que los factores están integrados por dos o más niveles o grupos, con el fin de averiguar entre cuáles existen diferencias significativas, se utilizó Scheffé como prueba de 
contraste post hoc, ya que los distintos grupos estaban formados por un número diferente de participantes. En todos los contrastes efectuados se asumió un nivel de significación de 0.05.

\section{Resultados}

Identificación de perfiles de regulación emocional

Para el establecimiento de los diferentes conglomerados se observó, en primer lugar, la existencia de la solución antes de las diez interacciones predeterminadas. En segundo lugar, además del criterio de convergencia estadística, para la elección del modelo se consideró su ajuste teórico, de modo que la solución más adecuada fue la formada por tres conglomerados. Las soluciones alternativas de cuatro y cinco grupos fueron muy similares, generando únicamente una mayor dispersión de los sujetos del grupo mixto sin aportar información relevante acerca de la combinación de las diferentes dimensiones. De esta forma, tal y como se muestra en la Figura 1, se definieron tres perfiles de regulación emocional, cada uno de ellos caracterizado por combinaciones diferentes de las cuatro dimensiones emocionales analizadas.

\section{Figura 1}

Perfiles de regulación emocional obtenidos por el análisis clúster.

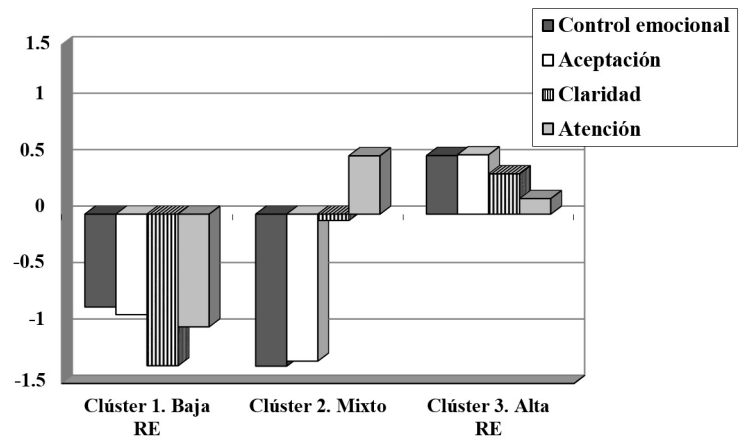

El primero de los clústeres se caracteriza por puntuaciones bajas en todas las dimensiones medidas. Se podría denominar, por tanto, como de "baja regulación emocional". Este primer grupo está integrado por 84 sujetos. El segundo de los conglomerados se caracteriza por altas puntuaciones en atención emocional y bajas puntuaciones en aceptación y control emocional. Las diferencias con el anterior radican en las elevadas puntuaciones en atención emocional y en un nivel prácticamente medio de claridad emocional por lo que se podría denominar como "grupo mixto", formado por 76 sujetos. El tercero de los conglomerados, que es claramente el más numeroso (está constituido por 327 sujetos, que corresponden al $66 \%$ de la muestra), se caracteriza por puntuaciones por encima de la media en todas las dimensiones; se podría denominar como de "alta regulación emocional".

Diferencias intergrupos en el uso de estrategias de afrontamiento

El ANOVA realizado puso de manifiesto que la diferencia en el uso de estrategias de afrontamiento activo entre los perfiles de regulación emocional identificados es estadísticamente significativa (Tabla 1). Concretamente, se observan diferencias entre los grupos en el factor reevaluación positiva $F_{(2}$, $484)=22, p<0.001$; en búsqueda de apoyo $F_{(2,484)}=16.85, p<0.001$ y planificación $F$ $(2,484)=8.69, p<0.001$. Se puede observar que el clúster 3 (alta regulación emocional) es el que obtiene puntuaciones más elevadas en el uso de las tres estrategias de afrontamiento activo evaluadas (reevaluación positiva, búsqueda de apoyo y planificación), seguido por el clúster 2 (grupo mixto) y, por último, el clúster 1, de baja regulación emocional, es el grupo que menores puntuaciones obtiene en las tres estrategias de afrontamiento (Tabla 1). 
Tabla 1

Análisis de la varianza en el uso de estrategias de afrontamiento en relación a los perfiles de regulación emocional

\begin{tabular}{llrrrr}
\hline $\begin{array}{l}\text { Estrategias de } \\
\text { Afrontamiento }\end{array}$ & $\begin{array}{l}\text { Perfiles de } \\
\text { Regulación } \\
\text { Emocional }\end{array}$ & $M$ & $D E$ & $F$ & $p$ \\
\hline Reevaluación & Baja & 2.51 & 0.75 & & \\
positiva & Mixta & 2.6 & 0.87 & 22 & 0.001 \\
\hline \multirow{2}{*}{ Búsqueda de } & Alta & 3.08 & 0.82 & & \\
Baja & 2.85 & 0.73 & & \\
& Mixta & 3.28 & 0.9 & 16.85 & 0.001 \\
& Alta & 3.43 & 0.83 & & \\
\hline \multirow{2}{*}{ Planificación } & Baja & 2.65 & 0.78 & & \\
& Mixta & 2.81 & 0.84 & 8.69 & 0.001 \\
& Alta & 3.04 & 0.81 & & \\
\hline
\end{tabular}

La comparación de las diferencias entre los diferentes perfiles de regulación emocional en el uso de estrategias de afrontamiento activo aportadas por el análisis post hoc (Tabla 2), se encuentra que el perfil de alta regulación emocional difiere significativamente del grupo de baja regulación emocional en las tres estrategias medidas; concretamente, en el uso del grupo de las estrategias de afrontamiento de reevaluación positiva, de búsqueda de apoyo y de la estrategia de planificación. Por su parte, al comparar el grupo 2 (mixto) con el grupo de alta regulación emocional, solo se observan diferencias significativas en el uso de la estrategia de reevaluación positiva. Por su parte, los grupos mixto y de baja regulación emocional difieren significativamente en el uso de la estrategia de búsqueda de apoyo (Tabla 2).

Tabla 2

Contrastes a posteriori (Scheffé) entre los perfiles de regulación emocional en relación al uso de estrategias de afrontamiento

\begin{tabular}{llrr}
\hline $\begin{array}{l}\text { Estrategias de } \\
\text { Afrontamiento }\end{array}$ & $\begin{array}{l}\text { Perfiles de } \\
\text { Regulación } \\
\text { Emocional }\end{array}$ & $t$ & \multicolumn{1}{c}{$p$} \\
\hline Reevaluación & Baja vs. Mixta & -0.088 & 0.795 \\
positiva & Mixta vs. Alta & -0.474 & $<0.001$ \\
& Baja vs. Alta & -0.562 & $<0.001$ \\
\hline \multirow{2}{*}{ Búsqueda de } & Baja vs. Mixta & -0.431 & 0.004 \\
apoyo & Mixta vs. Alta & -0.105 & 0.35 \\
& Baja vs. Alta & -0.583 & $<0.001$ \\
\hline \multirow{3}{*}{ Planificación } & Baja vs. Mixta & -0.163 & 0.44 \\
& Mixta vs. Alta & -0.221 & 0.096 \\
& Baja vs. Alta & -0.384 & $<0.001$ \\
\hline
\end{tabular}

\section{Discusión y conclusiones}

En el trabajo se plantearon dos objetivos relacionados. El primero intentó identificar las distintas combinaciones de regulación emocional (perfiles de regulación emocional) en la muestra de estudiantes universitarios de fisioterapia. Una vez determinados estos perfiles, interesaba comprobar la existencia de diferencias significativas entre los grupos obtenidos, en función del uso de estrategias de afrontamiento activo empleado por cada uno.

Los resultados ponen de manifiesto la existencia de perfiles con diferentes combinaciones de las dimensiones experiencial y estratégica de la regulación emocional. En concreto, se identificaron tres grupos: el primero caracterizado por una baja regulación emocional (bajas puntuaciones en las cuatro dimensiones medidas), el segundo con una alta atención emocional y bajas puntuaciones en aceptación y control emocional (grupo mixto) y finalmente, el tercero, que engloba alrededor de las dos terceras partes del total de los estudiantes de la muestra, caracterizado por una alta regulación emocional. Por tanto, se puede afirmar que, como grupo, los estudiantes universitarios de fisioterapia estudiados presentan un elevado nivel de regulación emocional, lo que es coherente con los resultados informados por Souto-Gestal (2014). Por el contrario, solo alrededor del $17 \%$ de los estudiantes se incluyen dentro del grupo de baja regulación emocional.

La caracterización de los perfiles de regulación emocional obtenidos podría explicarse por el hecho de que se trata de estudiantes de nivel universitario, en su mayoría con una elevada motivación hacia el servicio y la asistencia a otras personas en situaciones de discapacidad y dependencia, en la que los factores emocionales y las necesidades de percepción, comprensión y manejo de las emociones, tanto propias como ajenas, se encuentran siempre presentes.

En consecuencia, mientras el grupo de alta regulación emocional utiliza los componentes tanto experiencial como estratégico de la regulación emocional, siendo capaces de aceptar las emociones, controlarlas eficazmente y utilizar 
adaptativamente las estrategias de regulación, los estudiantes clasificados dentro del grupo de baja regulación emocional presentarían limitaciones en estas dimensiones. Por su parte, los estudiantes caracterizados con el perfil del grupo mixto presentan niveles de atención elevados, aunque, a diferencia del grupo de alta regulación, muestran valores de aceptación y control emocional muy reducidos. En comparación con el grupo de baja regulación emocional, el grupo mixto presenta puntuaciones de claridad emocional más cercanos a la media. Esta mayor comprensión sobre las emociones ha sido relacionada con el afrontamiento, especialmente el de tipo social e instrumental (Montes-Berges \& Augusto, 2007).

En todo caso, el análisis ha permitido establecer la presencia de perfiles diferenciales en relación con las diferentes habilidades que dan lugar a una adecuada regulación emocional. En este sentido, es necesario partir de la base de que una cosa es identificar y comprender las emociones y otra, ser capaz de controlarlas adecuadamente y utilizar estrategias de regulación adaptativas. Mientras que la atención y comprensión constituyen requisitos para la aceptación y control emocional, no lo determina. De este modo, se encuentran estudiantes que a pesar de no aceptar sus estados emocionales ni presentar habilidades para su gestión, muestran niveles elevados de atención emocional. En cuanto al segundo de los objetivos, los resultados ponen de manifiesto la existencia de diferencias estadísticamente significativas entre los grupos obtenidos en el uso informado de estrategias de afrontamiento activo.

Estos resultados aportan información que va más allá de la derivada de estudios anteriores, que indican la existencia de perfiles articulados en torno a las dimensiones de atención y claridad emocional (Diaz-Castela et al., 2013; Rey et al., 2011; Salguero et al., 2012), centradas, por tanto, en el componente experiencial de las emociones. Los citados trabajos señalan la existencia de perfiles que combinan de diferente manera los componentes experiencia y estratégico de la regulación emocional, apuntando a que esta combinación tiene efectos sobre cómo se gestionan las emociones y, como consecuencia, se da la adaptación más o menos eficazmente al contexto académico.

En línea con las investigaciones que vinculan regulación emocional y afrontamiento adaptativo (Downey et al., 2010; Martínez, Piqueras, \& Inglés, 2011; Mikolajczak et al., 2008; Petrides et al., 2007; Saklofske et al., 2007; Thomas et al., 2017, entre otros), el grupo de alta regulación emocional se diferencia significativamente del de baja regulación en el mayor uso de las tres estrategias de afrontamiento activo medidas (reevaluación positiva, búsqueda de apoyo y planificación). Las diferencias se producen tanto en el uso de las estrategias de afrontamiento cognitivas como comportamentales (incluyendo aquí la búsqueda de apoyo social). Con respecto al grupo mixto, las diferencias solo aparecen en el uso de la estrategia de reevaluación positiva. Ello podría explicarse por el hecho de que se conoce que el afrontamiento basado en la reevaluación positiva intensifica el proceso de regulación emocional (John \& Gross, 2007). En general, puede afirmarse que estos estudiantes afrontarían, pues, de forma más eficaz y adaptativa las situaciones estresantes derivadas de la vida académica.

Los estudiantes del grupo mixto y de baja regulación emocional difieren significativamente en el mayor uso informado que el grupo mixto hace de la estrategia de búsqueda de apoyo. Se podría afirmar que los grupos con mayores niveles de regulación emocional utilizan significativamente más esta estrategia de búsqueda de apoyo. Estos resultados serían concordantes, en líneas generales, con los obtenidos por Gázquez et al. (2015), si bien estos se obtuvieron en una muestra de adolescentes, donde solo el grupo de baja inteligencia emocional (lo que incluye baja regulación emocional) utilizó en menor medida conductas facilitadoras de la interacción social. Asimismo, también serían coherentes con los resultados de otros trabajos (Jiménez \& LópezZafra, 2011; Palomera, Salguero, \& RuizAranda, 2012; Salguero, Fernández-Berrocal, Ruíz-Aranda, Castillo, \& Palomera, 2011), en los 
cuales se muestra como las personas con mayores habilidades para percibir, comprender y/o regular sus emociones manifiestan una mayor calidad en sus relaciones interpersonales, caracterizadas por el apoyo, entre otras dimensiones sociales.

En relación con el grupo mixto, resulta importante destacar que la literatura ha puesto cierto énfasis en las personas que prestan una excesiva atención a sus emociones, es decir, que tienden a supervisar de forma continua cualquier evolución de su estado emocional. Esta característica podría resultar especialmente desadaptativa cuando los elevados niveles de atención emocional no se acompañan de la discriminación de las causas, razones y consecuencias (en otras palabras, de claridad o comprensión emocional). Estas personas corren el peligro de verse envueltas en una espiral emocional o círculo vicioso que, en lugar de aliviar su estado de ánimo perpetúa un estado anímico negativo a través de estrategias rumiativas, evitativas o impulsivas (Augusto \& López-Zafra, 2010). En relación con el empleo de estrategias de afrontamiento, este hecho no ha podido ser contrastado; si bien es cierto que el grupo mixto, además de caracterizarse por elevados niveles de atención, presentó una claridad muy próxima a la media. Futuros estudios deben indagar sobre la presencia de un perfil mixto más claramente definido por alta atención y baja claridad emocional y explorar su asociación con diferentes tipos de afrontamiento del estrés académico.

A pesar de las limitaciones del presente estudio, es posible señalar que los resultados obtenidos son novedosos, además de relevantes, pues ponen de manifiesto cómo las distintas combinaciones de los componentes de la regulación emocional afectan la manera en que se afronta el estrés. Contribuyen, por tanto, a conocer la relación entre regulación emocional y el afrontamiento del estrés académico, profundizando en el papel que las dimensiones personales desempeñan en la forma de afrontar las situaciones de amenaza o riesgo. La habilidad para gestionar adecuadamente las emociones propicia una valoración y un afrontamiento más adaptativo de los estímulos estresantes (Brougham, Zail, Mendoza, \& Miller, 2009).

Asimismo, los resultados de la presente investigación aportan información apreciable para el diseño de programas que permitan el entrenamiento en habilidades de manejo de las emociones en estudiantes universitarios, para prevenir la aparición de estrés, dotándolos de los recursos intrapsíquicos necesarios para afrontar con garantías las demandas académicas y el manejo del estrés (Brougham et al., 2009). En esta línea, Stoller, Taylor y Farver (2013) señalan la necesidad de incluir la formación en competencias emocionales dentro de los programas de formación en ciencias de la salud, con la finalidad de dotar a los estudiantes de destrezas que necesitarán en su futuro desarrollo profesional.

Hasta el momento, algunos de estos programas se han centrado en el incremento de competencias emocionales, mostrando buenos resultados (Cherry, Fletcher, O'Sullivan, \& Shaw, 2012; Nelis, Quoidbach, Mikolajczak, \& Hansenne, 2009), pero sin relacionarlos directamente con los niveles de estrés académico ni con las estrategias de afrontamiento empleadas. Los resultados que aporta este trabajo deben servir para el desarrollo y mejora de programas que combinen los componentes experiencial y estratégico de la regulación emocional, integrándolos como un todo. De esta manera, el estudio sobre un mejor conocimiento de las emociones debe combinarse con la aceptación y con un eficaz uso de estrategias de regulación emocional.

\section{Referencias}

Augusto, J. M., \& López-Zafra, E. (2010). The impact of emotional intelligence on nursing: An overview. Psychology, 1, 50-58. http://dx.doi.org/10.4236/psych.201 0.11008

Austin, E. J., Saklofske, D. H., \& Mastoras, S. M. (2010). Emotional intelligence, coping and exam-related stress in Canadian undergraduate students. Australian Journal 
of Psychology, 62 (1), 42-50. http://dx.doi.or $\mathrm{g} / 10.1080 / 00049530903312899$

Bardeen, J. R., Fergus, T. A., \& Orcutt, H. K. (2012). An examination of the latent structure of the Difficulties in Emotion Regulation Scale. Journal of Psychopathology and Behavioral Assessment, 34(3), 382-392. http://dx.doi.org/10.1007/s 10862-012-9280-y

Birks, Y., McKendree, J., \& Watt, I. (2009). Emotional intelligence and perceived stress in healthcare students: A multiinstitutional, multi-professional survey. BMC Medical Education, 9(1), 61. http://dx .doi.org/10.1186/1472-6920-9-61

Brougham, R. R., Zail, C. M., Mendoza, C. M., \& Miller, J. R. (2009). Stress, sex differences, and coping strategies among college students. Current Psychology, 28(2), 85-97. http://dx.doi.org/10.1007/s12144-0 09-9047-0

Cabanach, R. G., Souto-Gestal, A., Fernández-Cervantes, R., \& Freire, C. (2011). Regulación emocional y burnout académico en estudiantes universitarios de fisioterapia. Revista de Investigación en Educación, 2(9), 7-18. Recuperado de http://www.academia.edu/15233054/Re gulaci\%C3\%B3n_emocional_y_burnout_a cad\%C3\%A9mico_en_estudiantes_univer sitarios_de_Fisioterapia

Cabanach, R. G., Souto-Gestal, A., \& Franco, V. (2016). Escala de Estresores Académicos para la evaluación de los estresores académicos en estudiantes universitarios [Stressor Academic Scale for the evaluation of academic stressors in undergraduates]. Revista Iberoamericana de Psicología y Salud, 7(2), 41-50. http://dx.doi.org/10.1016/j.rips .2016 .05 .001

Cabanach, R. G., Valle, A., Rodríguez, S., Piñeiro, I., \& Freire, C. (2010). Escala de afrontamiento del estrés académico (A-CEA). Revista Iberoamericana de Psicología y Salud, 1(1), 51-64. Recuperado de http://www.redalyc.org/pdf/2451/24511 6411005.pdf
Cherry, M. G., Fletcher, I., O'sullivan, H., \& Shaw, N. (2012). What impact do structured educational sessions to increase emotional intelligence have on medical students? [BEME Guide No. 17]. Medical Teacher, 34(1), 11-19. Recuperado de http://www.redalyc.org/pdf/2451/24511 6411005.pdf

Diaz-Castela, M., Hale, W. W., III., Muela, J. A., Espinosa-Fernández, L., Klimstra, T., \& Garcia-Lopez, L. J. (2013). The measurement of perceived emotional intelligence for Spanish adolescents with social anxiety disorder symptoms. Anales de Psicología, 29(2), 509-515. http://dx.doi.org /10.6018/analesps.29.2.144271

Doherty, E. M., \& Nugent, E. (2011). Personality factors and medical training: A review of the literature. Medical Education, 45(2), 132-140. http://dx.doi.org/10.1111/j.13652923.2010.03760.x

Downey, L. A., Johnston, P. J., Hansen, K., Birney, J., \& Stough, C. (2010). Investigating the mediating effects of emotional intelligence and coping on problem behaviours in adolescents. Australian Journal of Psychology, 62 (1), 20-29. http://dx.doi.org/1 $0.1080 / 00049530903312873$

Fernández-Berrocal, P., Extremera, N., \& Palomera, R. (2008). Emotional intelligence as a crucial mental ability on educational context. En A. Valle, J. C. Núñez, R. G. Cabanach, J. GonzálezPienda \& S. Rodríguez (Eds.), Handbook of instructional resources and their applications in the classroom (pp. 67-88). NuevaYork: Nova Science.

Fischer, J. L., Forthun, L. F., Pidcock, B. W., \& Dowd, D. A. (2007). Parent relationships, emotion regulation, psychosocial maturity and college student alcohol use problems. Journal of Youth and Adolescence, 36(7), 912-926. http://dx.doi.org/10.1007/s10964 -006-9126-6

Folkman, S., \& Moskowitz, J. T. (2004). Coping: Pitfalls and promise. Annual Review of Psychology, 55, 745-74. http://dx.doi.org/10 .1146/annurev.psych.55.090902.141456 
García-Fernández, J. M., Inglés, C. J., Vicent, M., Gonzálvez, C., Gómez-Núñez, M. I., \& Poveda-Serra, P. (2016). Perfeccionismo durante la infancia y la adolescencia. Análisis bibliométrico y temático (2004-2014) [Perfectionism during childhood and adolescence. Bibliometric and thematic analysis (2004-2014)]. Revista Iberoamericana de Psicología y Salud, 7(2), 79-88. http://dx.doi .org/10.1016/j.rips.2016.02.001

Gázquez, J. J., Pérez-Fuentes, M. C., DíazHerrero, Á., García-Fernández, J. M., \& Inglés, C. J. (2015). Perfiles de inteligencia emocional y conducta social en adolescentes españoles. Psicología Conductual, 23(1), 141-160. Recuperado de http://www.funveca.org/revista/pedidos/ product.php?id_product $=667$

Gibbons, C., Dempster, M., \& Moutray, M. (2011). Stress, coping and satisfaction in nursing students. Journal of Advanced Nursing, 67(3), 621-632. http://dx.doi.org/ 10.1111/j.1365-2648.2010.05495.x

Gratz, K. L., \& Roemer, L. (2004). Multidimensional assessment of emotion regulation and dysregulation: Development, factor structure, and initial validation of the Difficulties in Emotion Regulation Scale. Journal of Psychopathology and Behavioral Assessment, 26(1), 41-54. http://dx.doi.org/10.1023/B:J OBA.0000007455.08539.94

Hair, J. F., Anderson, R. E., Tatham, R. L., \& Black, W. C. (1998). Multivariate data analysis. Upper Saddle River, NJ: PrenticeHall.

Hernandez, M. B., Blavo, C., Hardigan, P. C., Perez, A. M., \& Hage, K. (2010). Differences in perceived stress, depression, and medical symptoms among medical, nursing, and physician assistant students: A latent class analysis. Annals of Behavioral Science and Medical Education, 16(1), 35-39. http://dx.doi.org/10.1007/BF03355116

Hervás, G., \& Jódar, R. (2008). Adaptación al castellano de la Escala de Dificultades en la Regulación Emocional. Clínica y Salud,
19(2), 139-156. Recuperado de http://psyc net.apa.org/record/2008-17752-001

Jiménez, M. I., \& López-Zafra, E. (2011). Actitudes sociales y adaptación social en adolescentes españoles: el papel de la inteligencia emocional percibida. Revista de Psicología Social, 26(1), 105-117. http://dx. doi.org/10.1174/021347411794078417

John, O. P., \& Gross, J. J. (2007). Individual differences in emotion regulation strategies: Links to global trait, dynamic, and social cognitive constructs. En J. J. Gross (Ed.), Handbook of emotion regulation (pp. 351-372). Nueva York: Guilford Press.

MacCann, C., Fogarty, G. J., Zeidner, M., \& Roberts, R. D. (2011). Coping mediates the relationship between emotional intelligence (EI) and academic achievement. Contemporary Educational Psychology, 36(1), 60-70. http://dx.doi.org/1 0.1016/j.cedpsych.2010.11.002

Marín, M., Robles, R., González-Forteza, C., \& Andrade, P. (2012). Propiedades psicométricas de la escala "Dificultades en la Regulación Emocional" en español (DERS-E) para adolescentes mexicanos. Salud Mental, 35(6), 521-526. Recuperado de http://www.inprf-cd.gob.mx/pdf/sm3506 /sm3506521.pdf

Martínez, A. E., Piqueras, J. A., \& Inglés, C. J. (2011). Relaciones entre inteligencia emocional y estrategias de afrontamiento ante el estrés. Revista Electrónica de Motivación y Emoción, 14(37), 1-24. Recuperado de http://reme.uji.es/articulos/ numero37/article6/article6.pdf

Medrano, L. A., \& Trógolo, M. (2014). Validación de la escala de dificultades en la regulación emocional en la población universitaria de Córdoba, Argentina. Universitas Psychologica, 13(4), 1345-1356. http://dx.doi.org/10.11144/Jav eriana.UPSY13-4.vedr

Mikolajczak, M., Nelis, D., Hansenne, M., \& Quoidbach, J. (2008). If you can regulate sadness, you can probably regulate shame: Associations between trait emotional intelligence, emotion regulation and 
coping efficiency across discrete emotions. Personality and Individual Differences, 44(6), 1356-1368. http://dx.doi.org/10.1016/j.pai d.2007.12.004

Mikolajczak, M., Roy, E., Luminet, O., Fillée, C., \& de Timary, P. (2007). The moderating impact of emotional intelligence on free cortisol responses to stress. Psychoneuroendocrinology, 32(8), 1000-1012. http://dx.doi.org/10.1016/j.psy neuen.2007.07.009

Montes-Berges, B., \& Augusto, J. M. (2007). Exploring the relationship between perceived emotional intelligence, coping, social support and mental health in nursing students. Journal of Psychiatric and Mental Health Nursing, 14(2), 163-171. http://dx.d oi.org/10.1111/j.1365-2850.2007.01059.x

Naidoo, S., \& Pau, A. (2008). Emotional intelligence and perceived stress. SADJ: Journal of the South African Dental Association, 63(3), 148-151. Recuperado de https://www.ncbi.nlm.nih.gov/pubmed/ 18689346

Nelis, D., Quoidbach, J., Mikolajczak, M., \& Hansenne, M. (2009). Increasing emotional intelligence: (How) is it possible? Personality and Individual Differences, 47(1), 36-41. http://dx.doi.org/10.1016/j.paid.200 9.01 .046

Palomera, R., Salguero, J. M., \& Ruiz-Aranda, D. (2012). La percepción emocional como predictor estable del ajuste psicosocial en la adolescencia. Revista Internacional Clínica $y$ de Salud, 20(1), 43-58. Recuperado de http://behavioralpsycho.com/product/in teligencia-emocional-y-aculturacion-2/

Pastor, D. A., Barron, K. E., Miller, B. J., \& Davis, S. L. (2007). A latent profile analysis of college student's achievement goal orientation. Contemporary Educational Psychology, 32 (1), 8-47. http://dx.doi.org/10 .1016/j.cedpsych.2006.10.003

Pau, A., Rowland, M. L., Naidoo, S., AbdulKadir, R., Makrynika, E., Moraru, R., ... \& Croucher, R. (2007). Emotional intelligence and perceived stress in dental undergraduates: A multinational survey.
Journal of Dental Education, 71(2), 197-204. Recuperado de http://www.jdentaled.org/c ontent/71/2/197

Petrides, K. V., Pérez-González, J. C., \& Furnham, A. (2007). On the criterion and incremental validity of trait emotional intelligence. Cognition and Emotion, 21(1), 26-55. http://dx.doi.org/10.1080/02699930 601038912

Por, J., Barriball, L., Fitzpatrick, J., \& Roberts, J. (2011). Emotional intelligence: Its relationship to stress, coping, well-being and professional performance in nursing students. Nurse Education Today, 31(8), 855-860. http://dx.doi.org/10.1016/j.nedt.2 010.12 .023

Rey, L., Extremera, N., \& Pena, M. (2011). Perceived emotional intelligence, selfesteem and life satisfaction in adolescents. Psychosocial Intervention, 20(2), 227-234. ht tp://dx.doi.org/10.5093/in2011v20n2a10

Saklofske, D. H., Austin, E. J., Galloway, J., \& Davidson, K. (2007). Individual difference correlates of health-related behaviours: Preliminary evidence for links between emotional intelligence and coping. Personality and Individual Differences, 42 (3), 491-502. http://dx.doi.org/10.1016/j.paid.2 006.08 .006

Salguero, J. M., Fernández-Berrocal, P., RuizAranda, D., Castillo, R., \& Palomera, R. (2011). Inteligencia emocional y ajuste psicosocial en la adolescencia: el papel de la percepción emocional. European Journal of Education and Psychology, 4(2), 143-152. ht tp://dx.doi.org/10.1989/ejep.v4i2.84

Salguero, J. M., Palomera, R., \& FernándezBerrocal, P. (2012). Perceived emotional intelligence as predictor of psychological adjustment in adolescents: A 1-year prospective study. European Journal of Psychology of Education, 27(1), 21-34. http: //dx.doi.org/10.1007/s10212-011-0063-8

Salovey, P., Bedell, B. T., Detweiler, J. B., \& Mayer, J. D. (2000). Current directions in emotional intelligence research. En M. Lewis \& J. M. Haviland-Jones (Eds.), 
Handbook of emotions (2.a ed., pp. 504-520). Nueva York: Guilford Press.

Salovey, P., Stroud, L. R., Woolery, A., \& Epel, E. S. (2002). Perceived emotional intelligence, stress reactivity, and symptom reports: Further explorations using the Trait Meta-Mood Scale. Psychology and Health, 17(5), 611-627. http://dx.doi.org/10.1080/ 08870440290025812

Skinner, E. A., \& Zimmer-Gembeck, M. (2007). The development of coping. Annual Review of Psychology, 58, 119-144. http://dx.doi.org/10.1146/annure v.psych.58.110405.085705

Souto-Gestal, A. (2014). Regulación emocional y estrés académico en estudiantes de fisioterapia (Tesis doctoral inédita). Departamento de Psicología Evolutiva y de la Educación, Universidad de A Coruña.

Stoller, J. K., Taylor, C. A., \& Farver, C. F. (2013). Emotional intelligence competencies provide a developmental curriculum for medical training. Medical Teacher, 35(3), 243-247. http://dx.doi.org/1 0.3109/0142159X.2012.737964

Thomas, C. L., Cassady, J. C., \& Heller, M. L. (2017). The influence of emotional intelligence, cognitive test anxiety, and coping strategies on undergraduate academic performance. Learning and Individual Differences, 55, 40-48. http://dx.d oi.org/10.1016/j.lindif.2017.03.001

Walsh, J. M., Feeney, C., Hussey, J., \& Donnellan, C. (2010). Sources of stress and psychological morbidity among undergraduate physiotherapy students. Physiotherapy, 96(3), 206-212. http://dx.doi .org/10.1016/j.physio.2010.01.005

\section{Notas}

* Artículo de investigación. Esta investigación fue financiada en el marco del Programa de Consolidación y Estructuración de Unidades de Investigación Competitivas y por la Red "Salud, Bienestar Físico y Psicológico" (Exp. 2014/038) de la Consellería de Educación e Ordenación Universitaria de la Xunta de Galicia. 